\title{
Cancer Prediction with Gene Expression Data
}

\author{
G Sivagamasundari ${ }^{1}$, Latha Parthiban ${ }^{2}$
}

\begin{abstract}
With continuous growth in technology and quantum of data, many data mining algorithms are developed that uses micro array data to classify the genes and expressions in normal and disease conditions. There are many clustering algorithms that help to classify the genes and there is conflict in large pool of genes and their expression characters. The proposed system takes the input from multiple sources produces associate storage, cluster the information and classify it.
\end{abstract}

Index Terms: Data mining, Gene expression data, Classification.

\section{INTRODUCTION}

Biological research includes sequences of genes and proteins, gene expressions, biological functions, pathways etc. There are several techniques that can generate large data of genes, proteins, and their expressions. Different data mining methods are used to predict the gene expression data, and various algorithms are used to predict the genes and their functions The microarray data of both normal and disease conditions have several conditions of data that use cluster ways and microarray knowledge to classify. The microarray data of both normal and disease conditions have several conditions of data that use cluster ways and microarray knowledge to classify the genes. Based on the literature and different statistical approaches, there are several clustering algorithms that are used to built gene expression data and classification, however little attention has been paid to uncertainty within the results obtained

To get a transparent image on the sight of a angle, a transparent cancer classification associate analysis system has to be pictured followed by a scientific approach to analyse international organic phenomenon that provides an optimized answer for the known drawback space. Molecular medical speciality provides a possibility of human cancer classification, however these tests don't seem to be wide applied as a result of characteristic molecular markers for many solid neoplasm save nevertheless to be known.

Biological research includes sequences of genes and proteins, gene expressions, biological functions, pathways etc. There are several techniques that can generate large data of genes, proteins, and their expressions. Different data mining methods are used to predict the gene expression data, and various algorithms are used to predict the genes and their functions The microarray data of both normal and disease conditions have several conditions of data that use cluster

Revised Manuscript Received on July 05, 2019.

G Sivagamasundari Research Scholar, School of Engineering and Technology, Pondicherry University, India

Latha Parthiban, Department of Computer Science, Pondicherry University CC, Puducherry, India Latha Parthiban, Department of Computer Science, Pondicherry University CC, Puducherry, India Second Author name, His Department Name, University/ College/ Organization Name, City Name, Country Name. ways and microarray knowledge to classify. The microarray data of both normal and disease conditions have several conditions of data that use cluster ways and microarray knowledge to classify the genes. Based on the literature and different statistical approaches, there are several clustering algorithms that are used to built gene expression data and classification, however little attention has been paid to uncertainty within the results obtained

To get a transparent image on the sight of a angle, a transparent cancer classification associate analysis system has to be pictured followed by a scientific approach to analyse international organic phenomenon that provides an optimized answer for the known drawback space. Molecular medical speciality provides a possibility of human cancer classification, however these tests don't seem to be wide applied as a result of characteristic molecular markers for many solid neoplasm save nevertheless to be known.

\section{BACKGROUND WORK}

Many researchers presented data clustering that discovers the similarity or dissimilarity between groups of items in a dataset. Automated weighted method for attribute selection, generate the initial cluster centers rather than random or user specified cluster centres. An initialization problem can be resolved by binary search based initialization method to initialize cluster points for k-means algorithm. $\mathrm{K}-\mathrm{Means}$ algorithm is simple, efficient and provides easy convergence. But it has drawbacks like initial cluster centres, stuck in local optima. Lack of knowledge, lack of universal method, and no information about number. of clusters.

Anirban Mukhopadhyay et al 2013 stated that data clustering is an important task of datasets, where a set of data is grouped on its similarities. But there occurs an optimization problem with the clusters[1]. To overcome this multiple genetic based algorithm is applied on clusters characteristics such as compactness, separation and connectivity. The proposed approach Interactive Genetic Algorithm methodology uses a set of objective functions to arrive with the best results. But biological information is taken into account for validating the clusters. .

In [3], the authors described the gene clustering with a valid proximity measure which is useful in achieving accurate results for microarray data. The approach proposed in [4] takes into account 16 proximity measures in 52 data sets for the clustering of genes using Intrinsic Biological Separation Ability (IBSA) methodology. Result proposes Rank-Magnitude correlation coefficient as a measure for cancer and YS1 for time-course experiments. Proximity measurement measures the similarity between two data objects or gene expression patterns. There is no proper advice for selecting a proper proximity measure for the

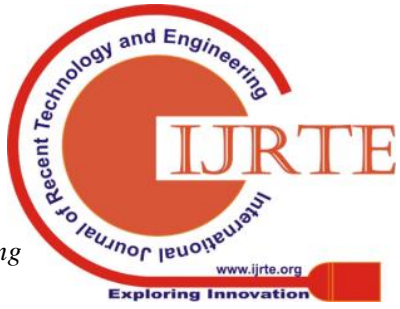


cluster, as it is very useful for the clustering of gene expression time course data.

.Balasundar I. Raju et.al 2013[2] proposed a new method called gene therapy is an experimental practice to treat or prevent disease using a person's genes. Ultrasound-mediated Delivery (USMD) methodology is used in clinical practice for safety and effectiveness. The USMD delivers the gene to the liver via tail vein site, using the process named sonoporation. This proves to be successful as the circulation time of Plasmid DNA is increased and the energy is delivered deep into the tissue.

Authors in [5] described the various tools that are required to integrate microarray gene expression (MAGE) data, as it is an important factor of gene-expression analysis. So the task is to identify the differences in batch execution effects.

Authors in [6-7] proved that cancer identification and classification tasks are important process during tumor discovery. Micro-array based gene expression proved to produce fruitful cancer identification results. The proposed approach uses support vector machines (SVM) as an iterative procedure and employs unlabled data and produces experiential results. But the situation is corrupted when introducing labeled data in the scene. The potential gene markers for each cancer subtype has been identified, which helps in successful cancer diagnosis. It manages an unlabeled gene expression data and achieved better empirical success .It is time consuming, expensive and tested with limited samples. In [8-9], the authors presented the DNA micro arrays and methods for gene ranking or gene expression data that includes T-score and ANOVA(Selection of important genes)

\section{EXPERIMENTAL RESULTS}

Microarray technology has made it possible to study the expression levels of thousands of genes simultaneously. In this work, real data collected from the hospitals of different patients have been used. The Patients data have been compared with the expert's documental data as shown by heat map solution. Proper Examination of gene expression data points to cancer identification and classification, that helps in proper treatment selection.

The implementation contains four stages: Data Set Input, Gene Knowledge Extraction, Ontological Mapping and Gene
Expression Design as shown in fig 2 to fig 5. Fig 6 shows the ontological mapping implementation, fig 7 the heat map, fig 8 the knowledge data

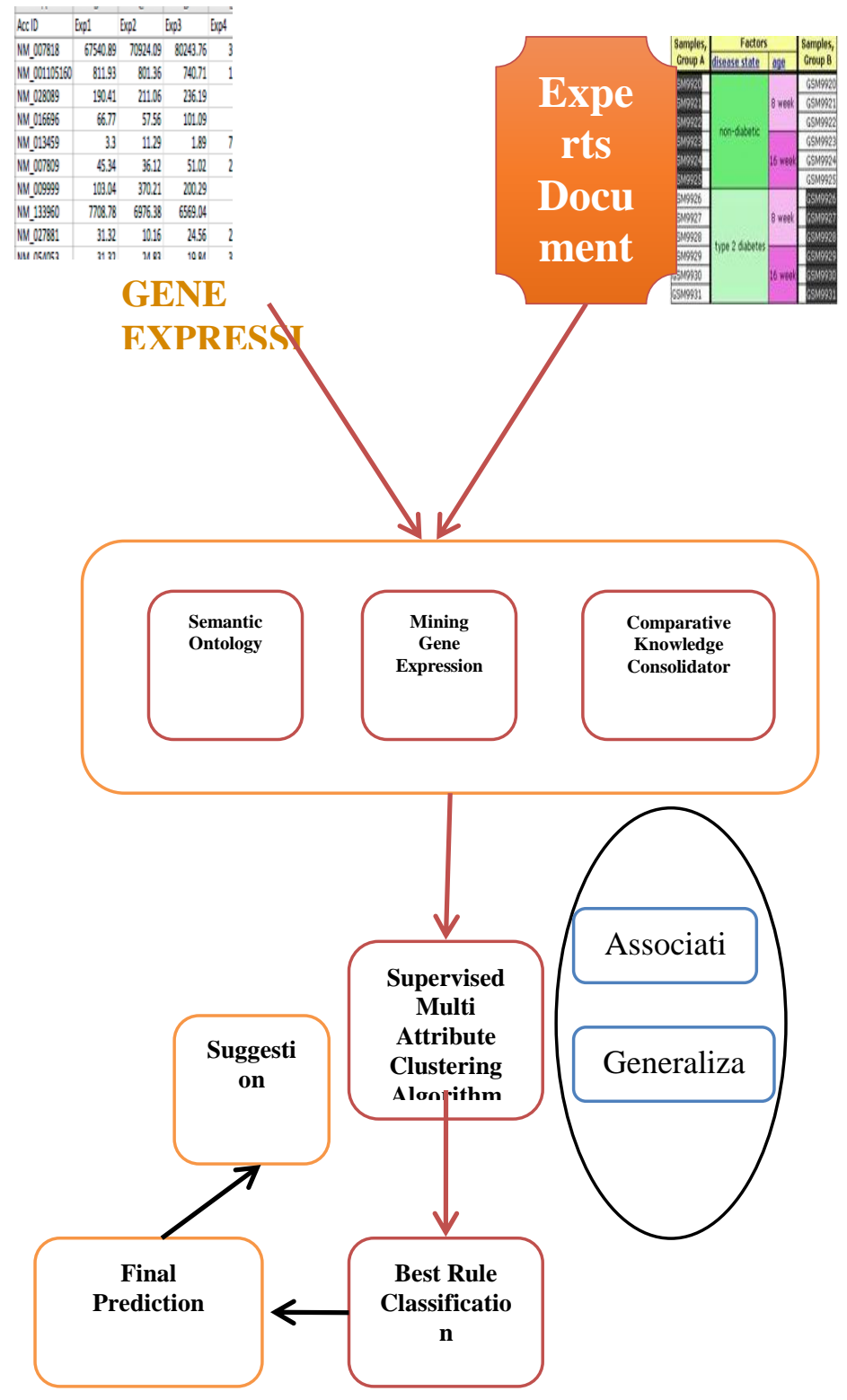

Fig 1 Architecture Diagram (KEOPS method)

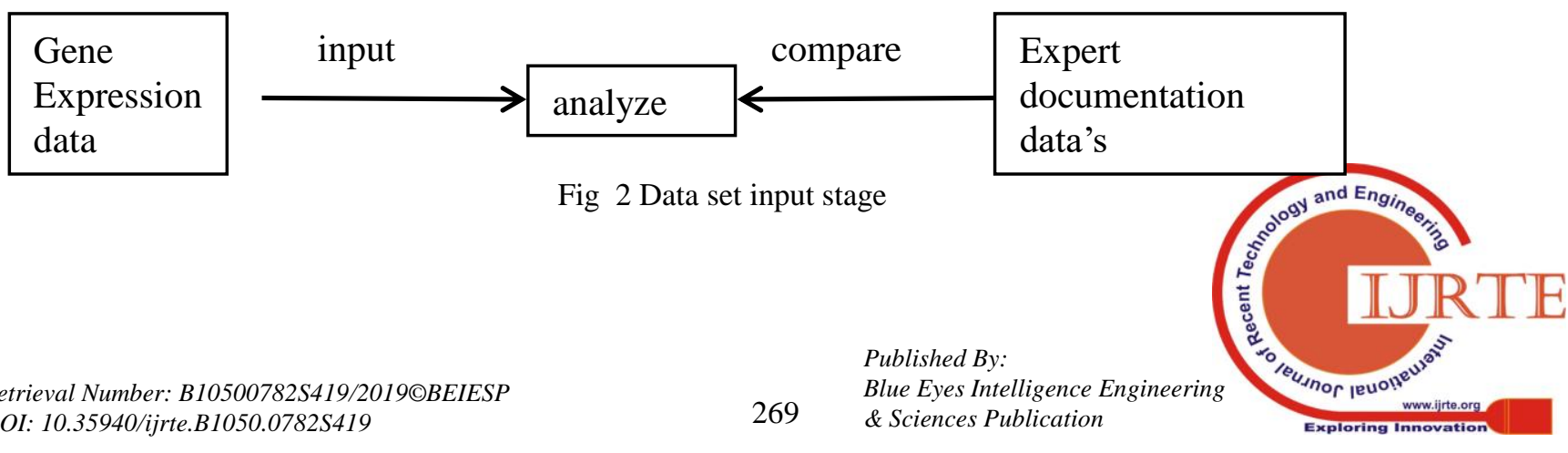


International Journal of Recent Technology and Engineering (IJRTE)

ISSN: 2277-3878, Volume-8 Issue-2S4, July 2019

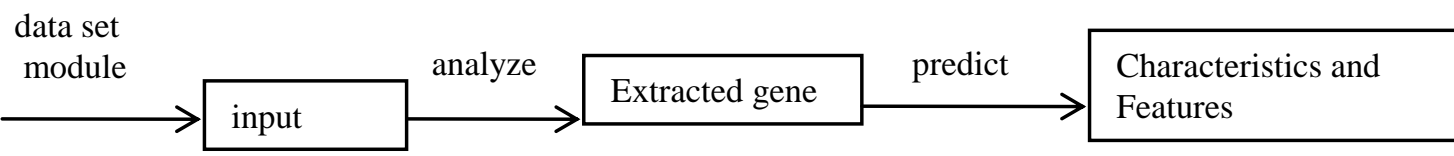

Fig 3 Gene Knowledge extraction

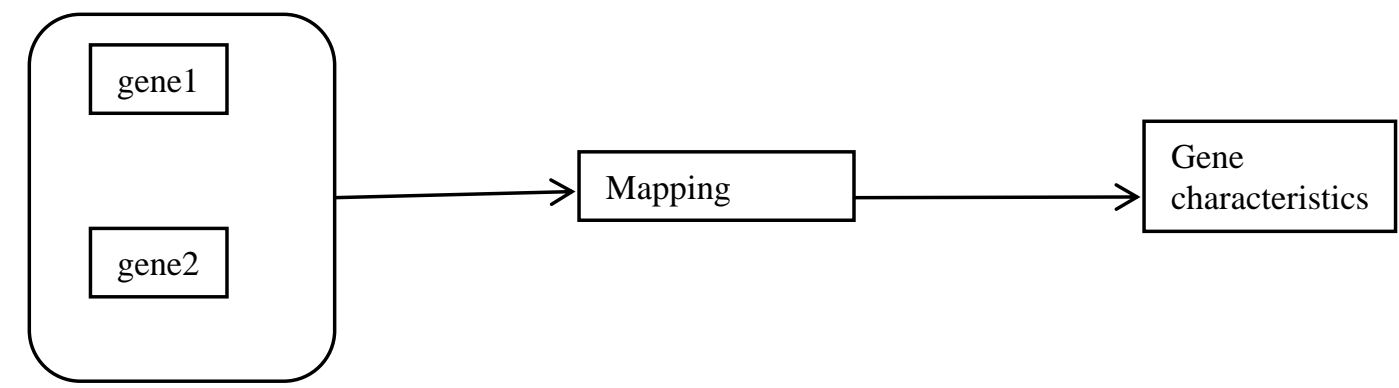

Fig 4 Ontological Mapping

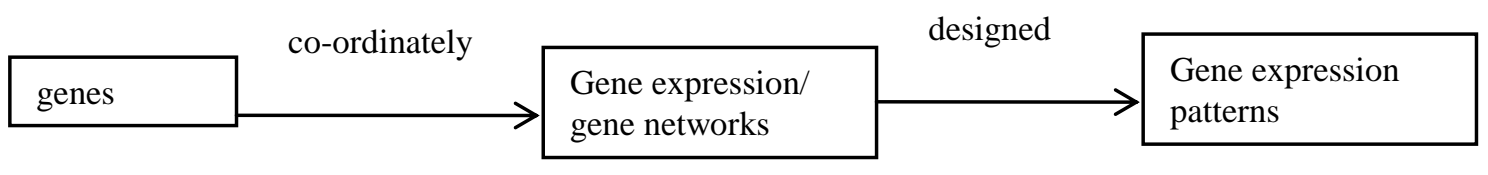

Fig 5 Gene Expression Design

\begin{tabular}{|c|c|c|c|c|c|c|c|c|c|}
\hline & ID & Gene title & Gene symbol & Gene ID & UniGene title & $\begin{array}{l}\text { UniGene } \\
\text { symbol }\end{array}$ & UniGene ID & $\begin{array}{l}\text { Nucleotide } \\
\text { Title }\end{array}$ & $\hat{\imath}$ \\
\hline \multirow[t]{10}{*}{ - } & 1007_s_at & discoidin domain ... & DDR1 & 780 & & & & Human receptort... & \\
\hline & 1053_at & replication factor ... & RFC2 & 5982 & & & & Human replicatio... & \\
\hline & 117_at & heat shock 70kD... & HSPAG & 3310 & & & & Human heatsho... & \\
\hline & 121_at & paired box 8 & PAX8 & 7849 & & & & H.sapiens Pax8 ... & \\
\hline & 1255___tt & guanylate cyclas... & GUCA1A & 2978 & & & & Homo sapiens gu... & \\
\hline & 1294_at & ubiquitin-like modi... & UBA7 & 7318 & & & & Homo sapiens ub... & \\
\hline & 1316_at & thyroid homone r... & THRA & 7067 & & & & Homo sapiens m... & \\
\hline & 1320_at & protein tyrosine p... & PTPN21 & 11099 & & & & H.sapiens mRNA... & \\
\hline & 1405___at & chemokine (C.C... & CCL5 & 6352 & & & & Human T cellspe... & \\
\hline & 1431 at & cytochrome P450... & CYP2E1 & 1571 & & & & Human cytochro... & 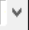 \\
\hline
\end{tabular}

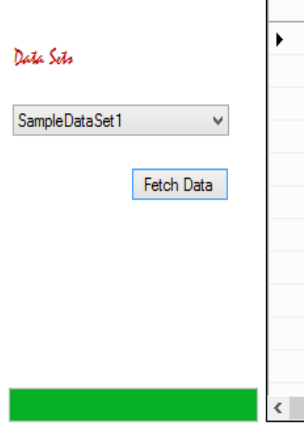

\begin{tabular}{|c|c|c|c|c|c|c|c|c|c|}
\hline & ID_REF & IDENTIFIER & GSM627133 & GSMG27216 & GSM627134 & GSM627151 & GSM627115 & GSM627087 & $\wedge$ \\
\hline \multirow[t]{11}{*}{ เ } & 231224_x_at & PRKAG2 & 35.8955 & 30.7351 & 32.9837 & 32.7659 & 37.144 & 38.6663 & \\
\hline & 240882_at & R85522 & 11.6371 & 10.5217 & 10.8537 & 11.8015 & 11.9516 & 13.0749 & \\
\hline & 1561849_at & PKD1L2 & 8.45343 & 8.46326 & 7.39276 & 7.33646 & 7.37014 & 7.52145 & \\
\hline & 1565746_at & LOC100132815 & 10.8116 & 11.1259 & 9.72156 & 8.65285 & 12.1602 & 10.7603 & \\
\hline & 1560853_x_at & ZNF826P & 16.0361 & 16.6499 & 14.5228 & 15.519 & 17.4264 & 16.0635 & \\
\hline & 230660_at & SERTAD4 & 12.4425 & 14.2759 & 12.7199 & 13.7253 & 12.7716 & 15.6567 & \\
\hline & 229708_at & TOR4A & 13.3051 & 13.8157 & 12.2562 & 19.5204 & 14.6117 & 15.015 & \\
\hline & 244781_x_at & R37682 & 8.12955 & 10.2744 & 8.59731 & 10.2062 & 9.19906 & 10.2366 & \\
\hline & 1554187_at & LOC554206 & 16.9199 & 15.6306 & 18.8628 & 17.3767 & 14.8512 & 15.015 & \\
\hline & 230021_at & TICRR & 25.4832 & 32.9972 & 27.267 & 26.6506 & 25.6564 & 21.8105 & \\
\hline & 238226_at & TMEM255B & 29.5791 & 26.6391 & 27.1048 & 25.821 & 27.9684 & 28.0039 & \\
\hline & mamam & Ta... & Anmen. & mans & raman & $m a n$ & man.. & $m a n$ & \\
\hline
\end{tabular}

Fig 6 Ontological mapping 


\section{Cancer Prediction with Gene Expresssion Data}

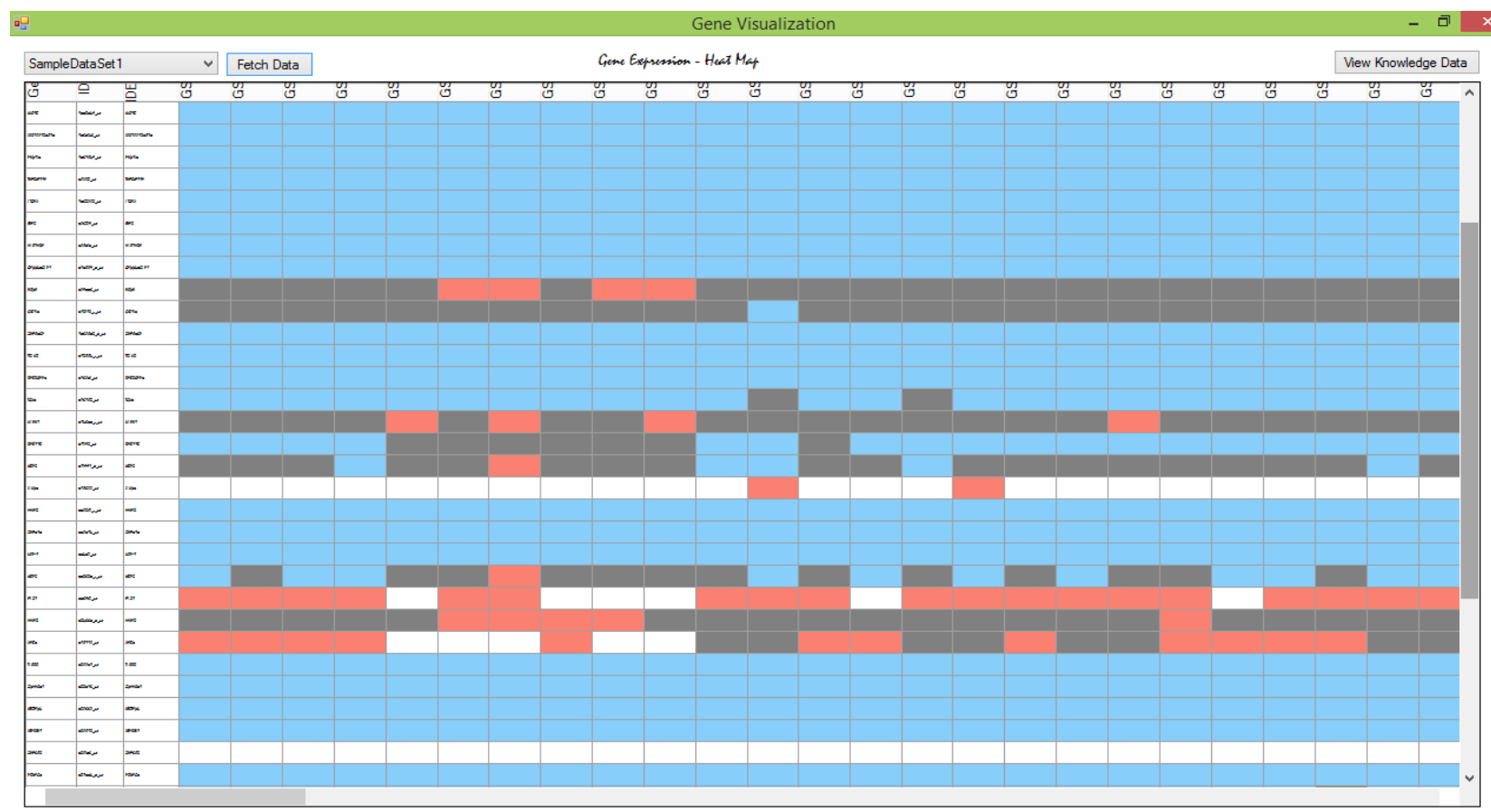

Fig 7 Heat map

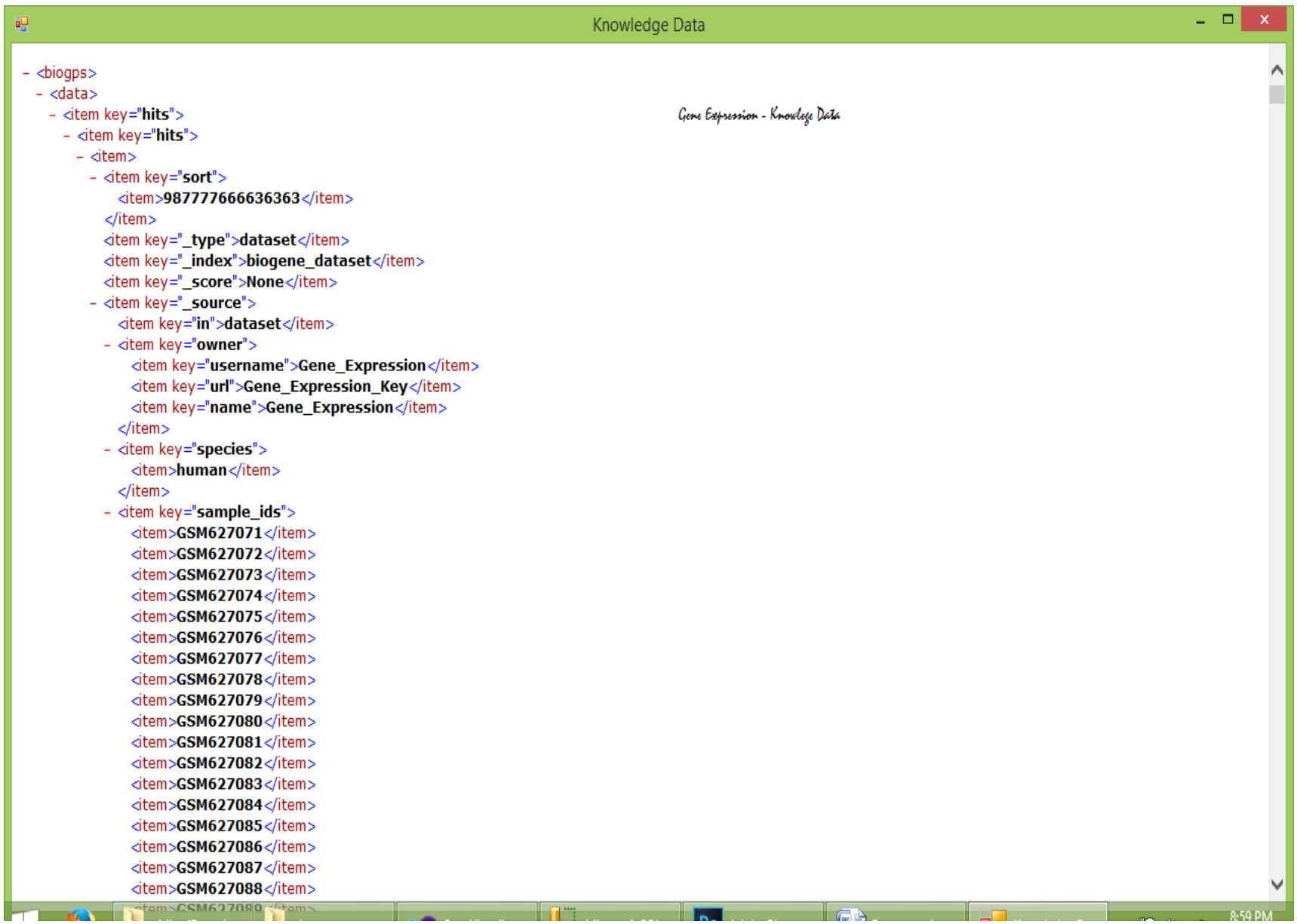

Fig 8 Knowledge data 
Table 1 Data set input

\begin{tabular}{|l|l|l|l|l|l|l|}
\hline ID_REF & IDENTIFIER & GSM627133 & GSM627216 & GSM627134 & GSM27151 & GSM27115 \\
\hline 231224_at & PRKAG2 & 35.8955 & 30.7351 & 32.9837 & 32.7659 & 37.144 \\
\hline 240882_at & R85522 & 11.6371 & 10.5217 & 10.8537 & 11.8015 & 11.9516 \\
\hline 1561849_at & PKD1L2 & 8.45343 & 8.46326 & 7.39276 & 7.33646 & 7.37014 \\
\hline 1565746_at & LOC100132815 & 10.8116 & 11.1259 & 9.72156 & 8.65285 & 12.1602 \\
\hline 1560853_at & ZNF826P & 16.0361 & 16.6499 & 14.5228 & 15.519 & 17.4264 \\
\hline 230660_at & SERTAD4 & 12.4425 & 14.2759 & 12.7199 & 13.7253 & 12.7716 \\
\hline 229708_at & TOR4A & 13.3051 & 13.8157 & 12.2562 & 19.5204 & 14.6117 \\
\hline 244781_at & R37682 & 8.12955 & 10.2744 & 8.59731 & 10.2062 & 9.19906 \\
\hline 1554187_at & LOC554206 & 16.9199 & 15.6306 & 18.8628 & 17.3767 & 14.8512 \\
\hline 230021_at & TICRR & 25.4832 & 32.9972 & 27.267 & 26.6506 & 25.6564 \\
\hline 238226_at & TMEM255B & 29.5791 & 26.6391 & 27.1048 & 25.821 & 27.9684 \\
\hline
\end{tabular}

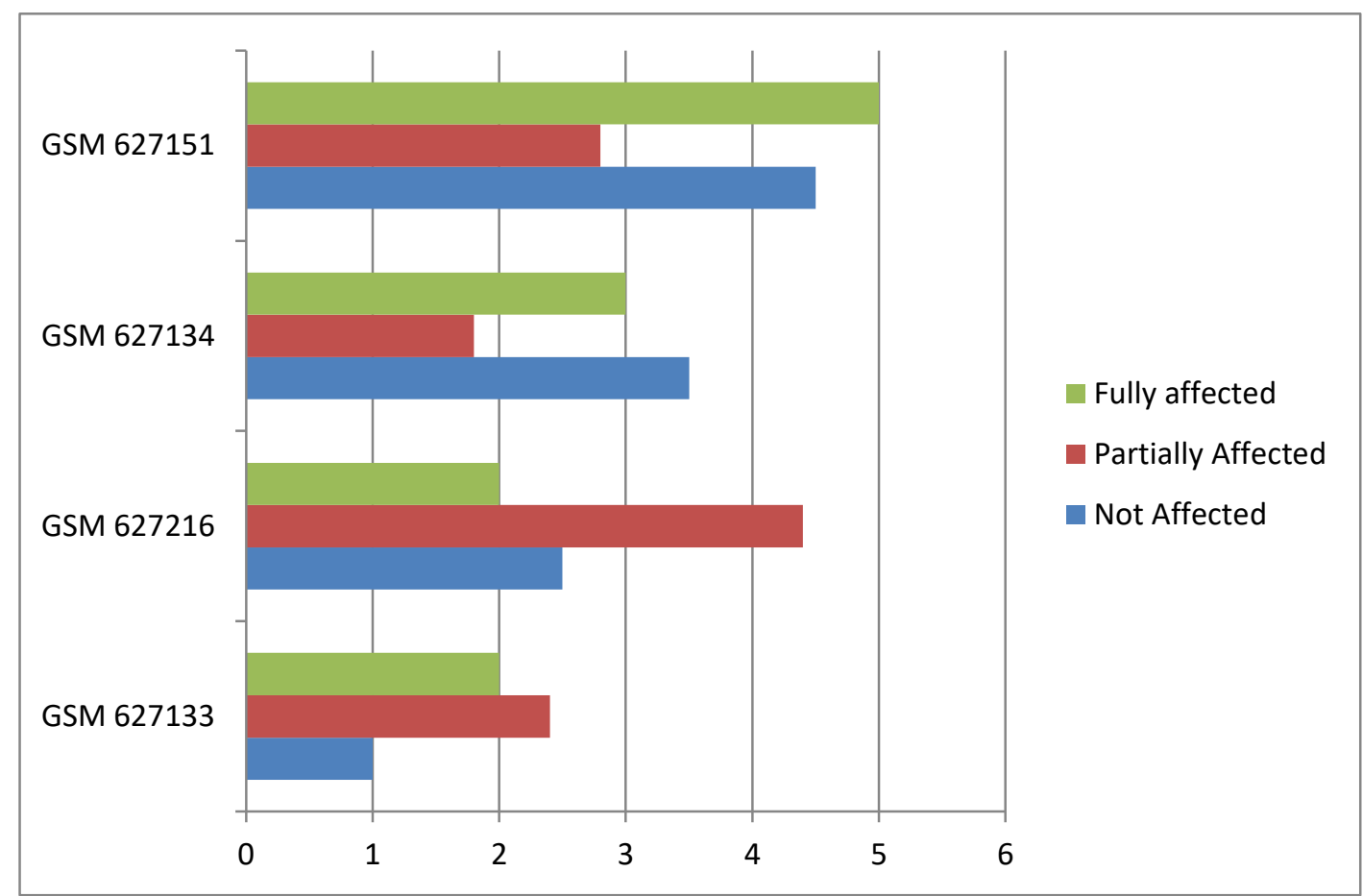

Fig 9 Heat map showing the affected levels

\section{CONCLUSIONS}

DNA Microarrays and heat maps are used to examine less gene and not entire genome. Prediction can be done easily when the heat map shows the affected levels. Sequencing in future can help in locating faulty genes as well as varied type of disease with better accuracy with the help of DNA chromosome sequence mutation. Where the mutation sequence can help to identify the cancer causing tissues.

\section{REFERENCES}

1. Anirban Mukhopadhyay,ujjwal maulik and sanghamitra Bandyopadhyay (2013) 'An Interactive approach to multiobjective clustering of gene expression patterns' IEEE transactions on biomedical engineering,volume. 60 No.1.
2. Balasundar I.Raju,Evgeniy Leyvi,Ralf seip,Shriram sethuraman,Andrew bird,Songtao Li,and Dwight koberi (2013) 'Enhanced Gene Expression of Systematically Administered Plasmid DNA in the Liver with Therapeutic Ultrasound and Micro bubbles' IEEE transactions on Ultrasonics,Ferroelectricals, and Frequency control, volume 60,No.1.

3. Bharathi And Dr.A.M.Natarajan (2010) 'Cancer Classification Of Bioinformatics Data Using ANOVA, International Journal Of Computer Theory And Engineering, Vol. 2, No. 3, 1793-8201.

4. Colin molter,Robin Duque,Hugues Bersini, and Ann Nowe(2013) 'GENESHIFT: A Nonparametric Approach for Integrating MicroArray Gene Expression Data Based on the Inner product as a distance Measure between the distributions of genes',IEEE/ACM transactions on computational biology and bioinformatics, volume 10,No.2.

5. Dimitris Maroulis, Dimitris Iakovidis, Ilias Flaounas and Stavros Karkanis(2006) 'A gene expression analysis system for medical diagnosis', IFIP International Federation for Published By:

Blue Eyes Intelligence Engineering

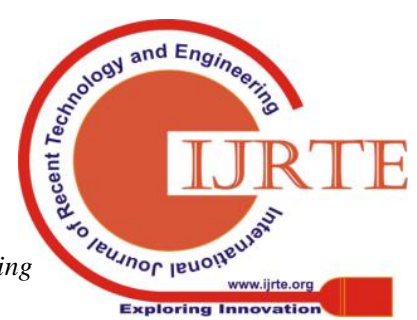


ISSN: 2277-3878, Volume-X, Issue-X, don’t delete Top \& Bottom Header, \& Fill up Manuscript details (It Page, Bottom, Left Side)

Information Processing Volume 4 No 2

6. Huimin Zhao (2007) 'A multi-objective genetic programming approach to developing Pareto optimal decision trees', -ELSEVIER decision support systems 43 809-826.

7. Jaskowik, Ricardo, J.G.B.Campello,and Ivan G.Costa(2013) 'Proximity Measures for Clustering Gene Expression Microarray data:A Validation Methodology and a Comparative Analysis' IEEE transactions on biology and bioinformatics, Volume 10,No.4

8. Rui Xu, Anagnostopoulos, G.C. And Wunsch, D.C.I.I(2007) 'Multiclass Cancer Classification Using Semi supervised Ellipsoid ARTMAP And Particle Swarm Optimization With Gene Expression Data' IEEE/ACM Transactions On Computational Biology And Bioinformatics, Volume.4, No.1, pp. 65-77.

9. Shaurya Jauhari ans S.A.M.Rizvi(2014) 'Mining Gene Expression Data focusing cancer Therapeutics:A Digest' IEEE transactions on computational biology and bioinformatics,Volume.11 No.3. 